\title{
Kinematic Jacobi Identity is a Residue Theorem: Geometry of Color-Kinematics Duality for Gauge and Gravity Amplitudes
}

\author{
Sebastian Mizera $\odot^{*}$ \\ Institute for Advanced Study, Einstein Drive, Princeton, New Jersey 08540, USA
}

(Received 10 December 2019; accepted 9 March 2020; published 7 April 2020)

\begin{abstract}
We give a geometric interpretation of color-kinematics duality between tree-level scattering amplitudes of gauge and gravity theories. Using their representation as intersection numbers we show how to obtain Bern-Carrasco-Johansson numerators in a constructive way as residues around boundaries of the moduli space. In this language the kinematic Jacobi identity between each triple of numerators is a residue theorem in disguise.
\end{abstract}

DOI: 10.1103/PhysRevLett.124.141601

Introduction.-Computation of scattering amplitudes in gravitational theories has traditionally posed a formidable task-even for tree-level processes-due to a proliferation of Feynman diagrams involved. This fact has changed with the introduction of color-kinematics duality [1,2] by Bern, Carrasco, and Johansson (BCJ), which provides a shortcut in computing gravitational observables by extracting the relevant information from gauge theory. It has since found applications in a spectrum of topics ranging from the study of ultraviolet properties of gravity [3-8], through the construction of classical solutions [9-16], to gravitational-wave physics [17-22].

Working at tree level, let us make the statement of colorkinematics duality more precise. Scattering amplitudes of $n$ gauge bosons can be expressed as

$$
\mathcal{A}_{n}^{\text {gauge }}=\sum_{\Gamma} \frac{n_{\Gamma} c_{\Gamma}}{\prod_{e \in \Gamma} p_{e}^{2}}
$$

where the sum goes over all $(2 n-5) ! !$ trivalent trees $\Gamma$ with propagators $p_{e}^{2}$ associated to each internal edge $e$ of $\Gamma$. Here $c_{\Gamma}$ denotes the color structure attached to each diagram, while $n_{\Gamma}$ is the remaining part of the numerator involving kinematic information such as contractions of momenta and polarization vectors.

Let us isolate triples of terms in Eq. (1) with graphs denoted by $\left(\Gamma_{s}, \Gamma_{t}, \Gamma_{u}\right)$ differing only by a single subdiagram, as in Fig. 1: Color structures associated to such triples satisfy the Lie algebra Jacobi identity, $c_{\Gamma_{s}}+c_{\Gamma_{t}}+$ $c_{\Gamma_{u}}=0$. Suppose that for every $\left(\Gamma_{s}, \Gamma_{t}, \Gamma_{u}\right)$ we enforce a similar condition on the kinematic numerators,

Published by the American Physical Society under the terms of the Creative Commons Attribution 4.0 International license. Further distribution of this work must maintain attribution to the author(s) and the published article's title, journal citation, and DOI. Funded by SCOAP ${ }^{3}$.

$$
n_{\Gamma_{s}}+n_{\Gamma_{t}}+n_{\Gamma_{u}}=0,
$$

known as the kinematic Jacobi identity. Since the numerators coming from Feynman diagram expansion do not naturally satisfy Eq. (2), it is typically a difficult task to bring them into such a form by reshuffling terms in Eq. (1). Assuming this can be done, BCJ proposed [1] that scattering amplitudes in gravity theory can be written, up to normalization, as

$$
\mathcal{A}_{n}^{\text {gravity }}=\sum_{\Gamma} \frac{n_{\Gamma} \tilde{n}_{\Gamma}}{\prod_{e \in \Gamma} p_{e}^{2}},
$$

where $n_{\Gamma}$ 's and $\tilde{n}_{\Gamma}$ 's are two (possibly distinct) sets of Jacobi-satisfying numerators. This statement is now proven [23] and can be extended to loop level [1,2,24-35], gauge and gravity theories with different supersymmetry and matter content [36-44], as well as various other theories [45-53]. Kinematic algebras leading to Eq. (2) have been investigated in Refs. [54-56]. For a comprehensive review of color-kinematics duality see Ref. [57].

At this stage one can ask if the kinematic Jacobi identity Eq. (2) has a geometric interpretation, and whether there exists a representation of scattering amplitudes that manifests this fact. These questions turn out to have a common answer, whose elucidation is the goal of this Letter.

It has recently emerged that a natural framework for addressing such problems is that of intersection theory [58]. It was previously used to provide a geometric interpretation

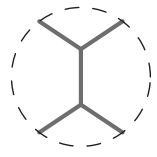

$\Gamma_{s}$

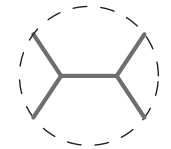

$\Gamma_{t}$

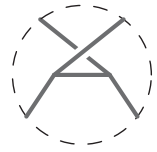

$\Gamma_{u}$
FIG. 1. Triples $\left(\Gamma_{s}, \Gamma_{t}, \Gamma_{u}\right)$ of diagrams differing by a subdiagram with four external legs. 
of Kawai-Lewellen-Tye (KLT) [59] relations between string- and field-theory amplitudes in terms of intersections of associahedra [60-62]; write down higher-loop monodromy and BCJ [1,63] relations for loop integrands [64]; understand precise conditions under which the low-energy limit of string-theory amplitudes localizes on scattering equations [58,62]; as well as give a new perspective on differential equations, dimensional recurrence relations, and integration-by-parts identities for multiloop Feynman integrals [65-68], among other applications [69-75]. At the same time this line of research unraveled connections between scattering amplitudes and more formal topics including Morse theory [58], Euler characteristics [62,65], Landau-Ginzburg models [68], and Yang-Baxter relations [62].

The central role in this theory is played by the so-called intersection numbers, which provide a geometric representation of tree-level amplitudes in various quantum field theories $[58,62]$. Selecting a theory amounts to specifying two differential forms, $\varphi_{-}$and $\varphi_{+}$, on the moduli space of Riemann spheres with $n$ punctures, $\mathcal{M}_{0, n}$. In the lowenergy limit intersection numbers are computed by [62]

$$
\sum_{\Gamma} \frac{\operatorname{Res}_{v_{\Gamma}}\left(\varphi_{-}\right) \operatorname{Res}_{v_{\Gamma}}\left(\varphi_{+}\right)}{\prod_{e \in \Gamma} p_{e}^{2}} .
$$

Here the sum is of exactly the same form as in Eqs. (1) and (3), and the role of numerators - both color and kinematic ones-is played by the residues around maximal-codimension boundaries of the moduli space, $v_{\Gamma}$, which are in one-to-one map with trivalent diagrams $\Gamma$.

We will prove that the numerators in Eq. (4) always satisfy the kinematic Jacobi identity Eq. (2) as a consequence of a residue theorem, thus providing a manifestly color-kinematics dual representation of amplitudes.

In this language the problem of finding numerators for various theories translates to different choices of $\varphi_{ \pm}$. After reviewing a known catalog of such forms for gauge and gravity theories we give explicit examples of computing Jacobi-satisfying numerators.

Boundaries and residues. - Let us briefly review the factorization structure of the moduli space $\mathcal{M}_{0, n}$ provided by its compactification [76]. When a subset $R$ of punctures collides on the Riemann sphere, the surface should be thought of as "bubbling" into two new spheres, where an emergent puncture $I$ separates the set $R$ from the complementary set $L$ (with sizes $2 \leq|L|,|R| \leq n-2$ ), see Fig. 2 . It is a codimension-one component of the boundary divisor $\partial \mathcal{M}_{0, n}$. We can make this procedure concrete on the level of differential forms. Take $\varphi$ to be a top (degree $n-3$ ) holomorphic form on $\mathcal{M}_{0, n}$, i.e., proportional to the $\mathrm{SL}(2, \mathbb{C})$-covariant measure

$$
d \mu_{n}=\left(z_{p}-z_{q}\right)\left(z_{q}-z_{r}\right)\left(z_{p}-z_{r}\right) \bigwedge_{\substack{i=1 \\ i \neq p, q, r}}^{n} d z_{i}
$$

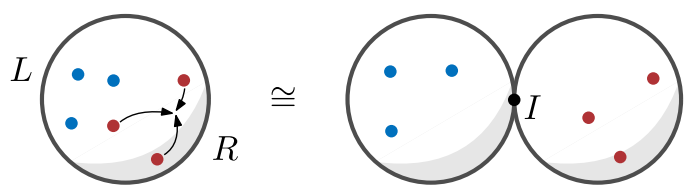

FIG. 2. Deligne-Mumford compactification of a codimensionone component of $\partial \mathcal{M}_{0, n}$.

where $\left(z_{p}, z_{q}, z_{r}\right)$ denote the positions of three arbitrary punctures fixed by the action of $\operatorname{SL}(2, \mathbb{C})$. For massless scattering we must require that $\varphi$ is invariant under $\mathrm{SL}(2, \mathbb{C})$ transformations $z_{i} \mapsto\left(A z_{i}+B\right) /\left(C z_{i}+D\right)$ with $A D-B C=1$ for all $z_{i}$ 's.

A standard way of modeling the above factorization is to embed the original sphere $\mathbb{C P}^{1}$ as a conic in $\mathbb{C P}^{2}$ with a new parameter $\epsilon$, such that it factors into $\mathbb{C P}^{1} \times \mathbb{C P}^{1}$ as $\epsilon \rightarrow 0$, see, e.g., Ref. [77]. In coordinates, we perform the change of variables

$$
z_{i}= \begin{cases}\epsilon / x_{i} & \text { for } i \in L, \\ y_{i} / \epsilon & \text { for } i \in R\end{cases}
$$

where $x_{i}$ 's and $y_{i}$ 's are positions of punctures on the new spheres with exactly two $x_{i}$ 's and two $y_{i}$ 's fixed. Since the boundary lies along $\left\{\epsilon^{2}=0\right\}$ we can simply take

$$
\operatorname{Res}_{\epsilon^{2}=0}(\varphi)=\varphi_{L} \wedge \varphi_{R},
$$

where $\varphi_{L}\left(x_{i}\right)$ and $\varphi_{R}\left(y_{i}\right)$ are now top (degree $|L|-2$ and $|R|-2$ ) holomorphic forms on the moduli spaces $\mathcal{M}_{0,|L|+1}$ and $\mathcal{M}_{0,|R|+1}$ of the left and right sphere, respectively. From the perspective of the particles on the left sphere the emergent puncture is at $x_{I}=0$, while from the right sphere it is at $y_{I}=0$. In the special case of two punctures colliding, i.e., $R=\left\{z_{i}, z_{j}\right\}$ the residue becomes simply $\operatorname{Res}_{z_{i}=z_{j}}(\varphi)=\varphi_{L}$ up to orientation. Intuitively, one might think of Eq. (7) as extracting a singular part in the operator product expansion between operators from the set $R$ being replaced by $I$ (or those from $L$ being replaced by $I$ from the other sphere's perspective).

Repeating this procedure exactly $n-3$ times one obtains maximal-codimension components (vertices) $v_{\Gamma}$ of $\partial \mathcal{M}_{0, n}$, which are in one-to-one map with trivalent graphs $\Gamma$, as all punctures are fixed by the action of $\operatorname{SL}(2, \mathbb{C})$, see, e.g.,

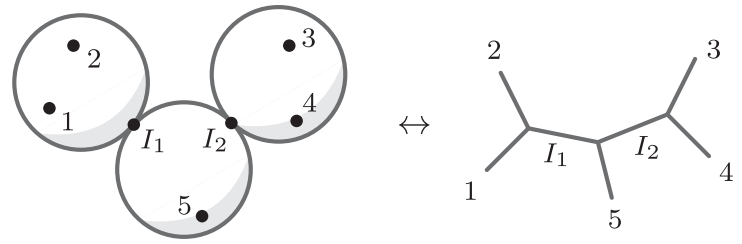

FIG. 3. Example of the map between maximal-codimension components of $\partial \mathcal{M}_{0, n}$ and trivalent trees. 


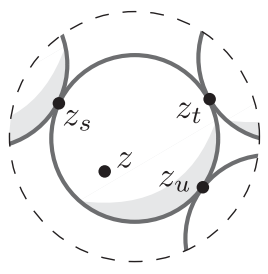

FIG. 4. The middle sphere with four punctures.

Fig. 3. The corresponding numerator $n_{\Gamma}=\operatorname{Res}_{v_{\Gamma}}(\varphi)$ is a function computed by applying Eq. (7) consecutively $n-3$ times.

There exists an alternative way of computing $\operatorname{Res}_{v_{\Gamma}}(\varphi)$, based on the dihedral extension of $\mathcal{M}_{0, n}$ employing cross-ratio coordinates suited for each $v_{\Gamma}$ [78], which is particularly useful for planar amplitudes, see, e.g., Refs. [62,69,79].

Kinematic Jacobi identity. - Let us consider the stage at which bubbling already happened $n-4$ times, i.e., when we are only one residue away from a trivalent factorization. It means there is exactly one sphere with four punctures, see Fig. 4. This leaves us with a one-form $\varphi_{M}$ on the moduli space of the "middle" sphere, which was computed as an $(n-4)$-fold residue of the original form $\varphi$. Let us call the unfixed puncture $z$ and the fixed ones $\left(z_{s}, z_{t}, z_{u}\right)$, such that $z$ colliding with $z_{i}$ leads to a trivalent graph $\Gamma_{i}$, as in Figure 1. By definition of the numerators entering Eq. (4) we have

$$
\begin{gathered}
n_{\Gamma_{s}}=\operatorname{Res}_{z=z_{s}}\left(\varphi_{M}\right), \quad n_{\Gamma_{t}}=\operatorname{Res}_{z=z_{t}}\left(\varphi_{M}\right), \\
n_{\Gamma_{u}}=\operatorname{Res}_{z=z_{u}}\left(\varphi_{M}\right),
\end{gathered}
$$

which are residues around the boundaries of the remaining moduli space, see Fig. 5. Since there are no other poles the residue theorem reads

$$
n_{\Gamma_{s}}+n_{\Gamma_{t}}+n_{\Gamma_{u}}=0,
$$

which is precisely the kinematic Jacobi identity Eq. (2). Given that we could have started with any configuration in Fig. 4, this identity is satisfied for all possible triples $\left(\Gamma_{s}, \Gamma_{t}, \Gamma_{u}\right)$. (The identity Eq. (9) means that for each triple

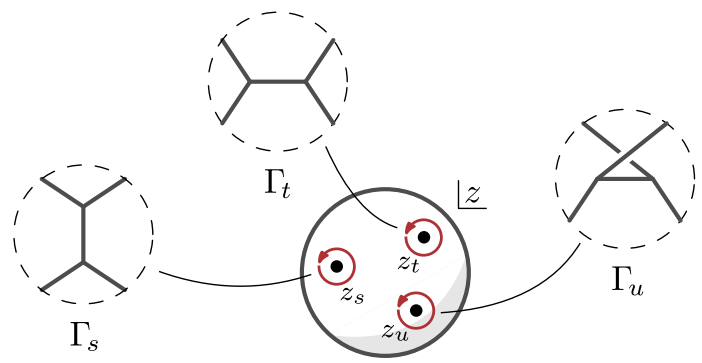

FIG. 5. Residue theorem on the moduli space of the middle sphere. only two out of three numerators are $\mathbb{Z}$ independent. One can ask how these relations combine for subdiagrams with $m \geq 4$ external legs by considering the "middle" sphere in Fig. 4 to have $m$ points. The results of Ref. [80] show that all residue theorems must reduce the number of $\mathbb{Z}$ independent numerators down to $\operatorname{dim} H^{m-3}\left(\mathcal{M}_{0, m}, \mathbb{Z}\right)=$ $(m-2)$ ! from the total of $(2 m-5) ! !$.)

Building blocks.-At this stage we have demonstrated that any rational form $\varphi$ on $\mathcal{M}_{0, n}$ leads to Jacobi-satisfying numerators, however, it does not yet mean that the resulting Eq. (4) is a scattering amplitude. We need to learn how to pick differential forms of physical relevance, which is a domain of intersection theory.

The first step is to realize that such forms should be really treated as elements of cohomology (equivalence) classes labeled by a \pm sign,

$$
\varphi_{ \pm} \sim \varphi_{ \pm}+(d \pm d W \wedge) \xi
$$

for any rational $(n-4)$-form $\xi$. Here $W$ is a potential given by

$$
W=\frac{1}{\Lambda^{2}} \sum_{i<j} 2 p_{i} \cdot p_{j} \log \left(z_{i}-z_{j}\right),
$$

with a mass scale $\Lambda$. This is precisely how $\varphi_{ \pm}$"know" about physics through the kinematic invariants $p_{i} \cdot p_{j}$. To distinguish them from ordinary differential forms we call $\varphi_{ \pm}$twisted forms. Their space is $(n-3)$ !-dimensional [81], in contrast with the space of ordinary forms, which is $(n-2)$ !-dimensional [80]. In order to make the statements below nontrivial we typically impose that twisted forms have no kinematic poles, which in turn implies that the numerators $n_{\Gamma}$ are local.

One can construct a bilinear of $\varphi_{-}$and $\varphi_{+}$called their intersection number, $\left\langle\varphi_{-} \mid \varphi_{+}\right\rangle_{d W}$, given by integrating the two forms over the moduli space. While such invariants have been known in mathematics for decades [82-84], only recently they were identified as representations of tree-level scattering amplitudes in various massive and massless quantum field theories in arbitrary space-time dimension [58], see Ref. [62] for a comprehensive introduction. We focus on massless external states, $p_{i}^{2}=0$, from now on.

There exists a catalog of twisted forms, which can be mixed and matched to compute different amplitudes [62]. For theories with color degrees of freedom $T^{c_{i}}$ we have

$\varphi_{ \pm}^{\text {color }}=d \mu_{n}\left(\frac{\operatorname{Tr}\left(T^{c_{1}} T^{c_{2}} \cdots T^{c_{n}}\right)}{\left(z_{1}-z_{2}\right)\left(z_{2}-z_{3}\right) \cdots\left(z_{n}-z_{1}\right)}+\right.$ perm. $)$,

where the symmetrization involves $(n-1)$ ! cyclic permutations (the definition is the same for both \pm ). By construction the associated numerator is precisely the color 
structure of a given diagram, i.e., $\operatorname{Res}_{v_{\Gamma}}\left(\varphi_{ \pm}^{\text {color }}\right)=c_{\Gamma}$, as in Eq. (1). For theories with polarization vectors $\varepsilon_{i}^{\mu}$ we can use

$$
\varphi_{ \pm}^{\text {gauge }}=d \mu_{n} \int \prod_{i=1}^{n} d \theta_{i} d \tilde{\theta}_{i} \frac{\theta_{k} \theta_{\ell}}{z_{k}-z_{\ell}} \exp \sum_{i \neq j} \Phi_{i j}
$$

(the choice of $k$ and $\ell$ is arbitrary) with

$\Phi_{i j}=-\frac{\theta_{i} \theta_{j} p_{i} \cdot p_{j}+\tilde{\theta}_{i} \tilde{\theta}_{j} \varepsilon_{i} \cdot \varepsilon_{j}+2\left(\theta_{i}-\theta_{j}\right) \tilde{\theta}_{i} \varepsilon_{i} \cdot p_{j}}{z_{i}-z_{j} \mp \Lambda^{2} \theta_{i} \theta_{j}}$.

For conciseness we wrote it in terms of Grassmann integrals over $\theta_{i}$ and $\tilde{\theta}_{i}$, which can be expanded as a degree- $\lfloor(n-2) / 2\rfloor$ polynomial in $\Lambda^{2}$ of Pfaffians [see, e.g., Eq. (4.8) in Ref. [62] ]. Similarly, we have the forms

$$
\varphi_{ \pm}^{\text {bosonic }}=d \mu_{n}( \pm \Lambda)^{n-2} \int \prod_{i=1}^{n} d \theta_{i} d \tilde{\theta}_{i} \exp \sum_{i \neq j} \Xi_{i j}
$$

where

$$
\Xi_{i j}= \pm \frac{1}{\Lambda} \frac{2 \theta_{j} \tilde{\theta}_{j} p_{i} \cdot \varepsilon_{j}}{z_{i}-z_{j}}+\frac{\theta_{i} \tilde{\theta}_{i} \theta_{j} \tilde{\theta}_{j} \varepsilon_{i} \cdot \varepsilon_{j}}{\left(z_{i}-z_{j}\right)^{2}} .
$$

Upon the identification $\Lambda^{2}=1 / \alpha^{\prime}$, Eqs. (13) and (15) are in fact the same objects as those in super- and bosonic string perturbation theory, respectively [85], but-surprisinglynow appear in a purely field-theoretic context.

A partial list of theories whose amplitudes are known to have an interpretation as intersection numbers is given in Table I [62]. Even though Eq. (13) depends on $\Lambda$, this dependence drops out from the resulting amplitudes in the first three cases (it is not true for the last two) $[62,86]$. Since amplitudes are written as bilinears in this representation, KLT relations between the above theories become simply a consequence of linear algebra. The total differential $0 \sim$ $(d \pm d W \wedge) \varphi_{ \pm, n-1}^{\text {color }}$ implies the fundamental BCJ relation [1], as an extension of the arguments in Ref. [87]. Twisted forms for states lying in the low-energy spectrum of string theory, such as those involving fermions or mixed EinsteinYang-Mills interactions, can be readily written down using

TABLE I. List of theories whose amplitudes $\left\langle\varphi_{-} \mid \varphi_{+}\right\rangle_{d W}$ are computed using twisted forms $\varphi_{ \pm}$.

\begin{tabular}{lll}
\hline \hline$\varphi_{-}$ & $\varphi_{+}$ & Theory \\
\hline$\varphi_{-}^{\text {color }}$ & $\varphi_{+}^{\text {color }}$ & Bi-adjoint scalar $[55,88]$ \\
$\varphi_{-}^{\text {color }}$ & $\varphi_{+}^{\text {gauge }}$ & Yang-Mills \\
$\varphi_{-}^{\text {gauge }}$ & $\varphi_{+}^{\text {gauge }}$ & Einstein gravity \\
$\varphi_{-}^{\text {color }}$ & $\varphi_{+}^{\text {bosonic }}$ & YM $+(D F)^{2}[42,89]$ \\
$\varphi_{-}^{\text {gauge }}$ & $\varphi_{+}^{\text {bosonic }}$ & Weyl-Einstein gravity $[42,89]$ \\
\hline \hline
\end{tabular}

the techniques discussed in Ref. [62], but we will not pursue it here.

Scattering amplitudes in such a representation can be computed exactly using recursion relations [62], however the resulting numerators do not come in a Jacobi-satisfying way. Instead, the localization formula Eq. (4) is known to arise as the $\Lambda^{0}$ order in the low-energy $(\Lambda \rightarrow \infty)$ expansion of intersection numbers [62],

$$
\left\langle\varphi_{-} \mid \varphi_{+}\right\rangle_{d W}=\sum_{\Gamma} \frac{\operatorname{Res}_{v_{\Gamma}}\left(\varphi_{-}\right) \operatorname{Res}_{v_{\Gamma}}\left(\varphi_{+}\right)}{\prod_{e \in \Gamma} p_{e}^{2}}+\mathcal{O}\left(\Lambda^{-2}\right),
$$

when $\varphi_{ \pm}$are independent of $\Lambda$. (In the massless limit $(\Lambda \rightarrow 0)$ intersection numbers have another localization formula on the so-called scattering equations, $d W=0$, which at the leading order $\Lambda^{0}$ gives the Cachazo-He-Yuan (CHY) $[88,90]$ formulation of massless amplitudes, see $[58,62]$ for details. Since Yang-Mills and Einstein gravity amplitudes are independent of $\Lambda$ to begin with, this limit is exact. Subleading corrections $\mathcal{O}\left(\Lambda^{2 p \geq 2}\right)$ are given by higher residue pairings $[68,91]$.) However, with the exception of Eq. (12), twisted forms given above are polynomials in $\Lambda^{2}$, which leads to mixing of different orders in Eq. (17). To consistently extract the leading order $\Lambda^{0}$ with Eq. (17) one needs to first remove the $\Lambda$ dependence from twisted forms by a repeated use of Eq. (10). Given that Yang-Mills and Einstein gravity amplitudes are independent of $\Lambda$, once this is done the terms of order $\mathcal{O}\left(\Lambda^{-2}\right)$ are not present and the numerators are exact.

Examples.-We proceed with two illustrative examples. In order to contain expressions within the margins of this Letter we focus on the case $n=4$, where amplitudes with color degrees of freedom take the form

$$
\mathcal{A}_{4}=\frac{n_{s} c_{s}}{s}+\frac{n_{t} c_{t}}{t}+\frac{n_{u} c_{u}}{u},
$$

with $s=\left(p_{1}+p_{2}\right)^{2}, t=\left(p_{2}+p_{3}\right)^{2}, u=\left(p_{1}+p_{3}\right)^{2}$ and a single triple. Fixing the punctures $\left(z_{1}, z_{2}, z_{3}\right)$ leaves us with a single coordinate $z_{4}$ on $\mathcal{M}_{0,4}$. Evaluating color numerators using Eq. (12) for $n=4$ amounts to computing the residues:

$$
\begin{aligned}
& c_{s}=\operatorname{Res}_{z_{4}=z_{3}}\left(\varphi_{-, 4}^{\text {color }}\right)=f^{c_{1} c_{2} b} f^{b c_{3} c_{4}}, \\
& c_{t}=\operatorname{Res}_{z_{4}=z_{1}}\left(\varphi_{-, 4}^{\text {color }}\right)=f^{c_{2} c_{3} b} f^{b c_{1} c_{4}}, \\
& c_{u}=\operatorname{Res}_{z_{4}=z_{2}}\left(\varphi_{-, 4}^{\text {color }}\right)=f^{c_{3} c_{1} b} f^{b c_{2} c_{4}},
\end{aligned}
$$

with the convention $f^{a b c}=\operatorname{Tr}\left(T^{a}\left[T^{b}, T^{c}\right]\right)$. In this case the residue theorem implies the usual Jacobi identity $c_{s}+c_{t}+c_{u}=0$.

Gauge theory.-Let us consider kinematic numerators in Yang-Mills theory. Choosing $(k, \ell)=(1,2)$ for $n=4$ the twisted form Eq. (13) becomes 


$$
\varphi_{+}^{\text {gauge }}=z_{13} z_{23}\left(\operatorname{Pf} \boldsymbol{\Psi}_{[12]}-4 \Lambda^{2} \frac{\varepsilon_{1} \cdot \varepsilon_{2} \varepsilon_{3} \cdot \varepsilon_{4}}{z_{12} z_{34}^{2}}\right) d z_{4} .
$$

Here $\boldsymbol{\Psi}_{[12]}$ is the matrix known from the CHY formalism [90] in the conventions of Ref. [62], with columns and rows 1,2 removed. In order to fix the issue with $\Lambda$ nonhomogeneity we use Eq. (10) with

$$
\xi=4 \Lambda^{2} \varepsilon_{1} \cdot \varepsilon_{2} \varepsilon_{3} \cdot \varepsilon_{4} \frac{z_{13} z_{24}}{z_{12} z_{34}}
$$

obtained by integrating minus the final term in Eq. (20). Adding $(d+d W \wedge) \xi$ to Eq. (20) gives us a form cohomologous to Eq (20), but independent of $\Lambda$ :

$$
\begin{aligned}
\varphi_{+, 4}^{\text {gauge }}= & z_{13} z_{23} P f \Psi_{[12]} d z_{4} \\
& +4 \varepsilon_{1} \cdot \varepsilon_{2} \varepsilon_{3} \cdot \varepsilon_{4}\left(\frac{t}{z_{41}}+\frac{u}{z_{42}}+\frac{s}{z_{43}}\right) \frac{z_{13} z_{24}}{z_{12} z_{34}} d z_{4} .
\end{aligned}
$$

Therefore the leading order in Eq. (17) computes the full Yang-Mills amplitude. Using this representation we find

$$
\begin{aligned}
n_{s}= & \operatorname{Res}_{z_{4}=z_{3}}\left(\varphi_{+, 4}^{\text {gauge }}\right) \\
= & 8 \varepsilon_{1, \mu} \varepsilon_{2, \nu} \varepsilon_{3, \rho} \varepsilon_{4, \tau}\left[p_{1} \cdot p_{2}\left(\eta^{\mu \rho} \eta^{\nu \tau}-\eta^{\mu \tau} \eta^{\nu \rho}\right)\right. \\
& -p_{2} \cdot p_{3} \eta^{\mu \nu} \eta^{\rho \tau}+\left(p_{1}^{\rho} p_{2}^{\tau}-p_{2}^{\rho} p_{1}^{\tau}\right) \eta^{\mu \nu}+p_{1}^{\nu} p_{3}^{\tau} \eta^{\mu \rho} \\
& \left.-p_{1}^{\nu} p_{4}^{\rho} \eta^{\mu \tau}-p_{2}^{\mu} p_{3}^{\tau} \eta^{\nu \rho}+p_{2}^{\mu} p_{4}^{\rho} \eta^{\nu \tau}+\left(p_{3}^{\mu} p_{4}^{\nu}-p_{4}^{\mu} p_{3}^{\nu}\right) \eta^{\rho \tau}\right],
\end{aligned}
$$

$$
\begin{aligned}
n_{t}= & \operatorname{Res}_{z_{4}=z_{1}}\left(\varphi_{+, 4}^{\text {gauge }}\right) \\
= & 8 \varepsilon_{1, \mu} \varepsilon_{2, \nu} \varepsilon_{3, \rho} \varepsilon_{4, \tau}\left[p_{1} \cdot p_{2} \eta^{\mu \tau} \eta^{\nu \rho}+p_{2} \cdot p_{3} \eta^{\mu \nu} \eta^{\rho \tau}\right. \\
& +p_{2}^{\rho} p_{1}^{\tau} \eta^{\mu \nu}-p_{3}^{\nu} p_{1}^{\tau} \eta^{\mu \rho}+\left(p_{1}^{\nu} p_{4}^{\rho}-p_{4}^{\nu} p_{1}^{\rho}\right) \eta^{\mu \tau} \\
& \left.+\left(p_{2}^{\mu} p_{3}^{\tau}-p_{3}^{\mu} p_{2}^{\tau}\right) \eta^{\nu \rho}-p_{4}^{\mu} p_{2}^{\rho} \eta^{\nu \tau}+p_{4}^{\mu} p_{3}^{\nu} \eta^{\rho \tau}\right] \\
n_{u}= & \operatorname{Res}_{z_{4}=z_{2}}\left(\varphi_{+, 4}^{\text {gauge }}\right) \\
= & 8 \varepsilon_{1, \mu} \varepsilon_{2, \nu} \varepsilon_{3, \rho} \varepsilon_{4, \tau}\left[-p_{1} \cdot p_{2} \eta^{\mu \rho} \eta^{\nu \tau}-p_{1}^{\rho} p_{2}^{\tau} \eta^{\mu \nu}\right. \\
& +\left(p_{3}^{\nu} p_{1}^{\tau}-p_{1}^{\nu} p_{3}^{\tau}\right) \eta^{\mu \rho}+p_{4}^{\nu} p_{1}^{\rho} \eta^{\mu \tau}+p_{3}^{\mu} p_{2}^{\tau} \eta^{\nu \rho} \\
& +\left(p_{4}^{\mu} p_{2}^{\rho}-p_{2}^{\mu} p_{4}^{\rho}\right) \eta^{\nu \tau}-p_{3}^{\mu} p_{4}^{\nu} \eta^{\rho \tau} .
\end{aligned}
$$

One can check that $n_{s}+n_{t}+n_{u}=0$ and the resulting amplitude [Eq. (18)] is gauge invariant. Scattering amplitude of four gravitons is obtained by replacing $c_{\Gamma} \rightarrow \tilde{n}_{\Gamma}$ (with $\tilde{\varepsilon}_{i}$ instead of $\varepsilon_{i}$ ) followed by a symmetrization of polarization tensors, $\varepsilon_{i}^{\mu \nu}=\varepsilon_{i}^{(\mu} \tilde{\varepsilon}_{i}^{\nu)}$.

Conclusion.-In this Letter we introduced a representation of tree-level scattering amplitudes that manifests colorkinematics duality. The problem of finding theories with Jacobi-satisfying numerators translates to a classification of twisted forms, which motivates further extension of their available catalog.

The amplitudes computed with Eq. (13) have a remarkable property of being $\Lambda$ independent, as expected for massless theories, despite the fact $\varphi_{ \pm}^{\text {gauge }}$ is not. On the other hand, it was previously shown that intersection numbers of logarithmic forms are independent of $\Lambda[58,84]$. Thus, one might suspect that once $\varphi_{ \pm}^{\text {gauge }}$ is brought into a $\Lambda$ independent form (perhaps using the algorithms of $[29,86,92-97])$ it would become logarithmic, as is the case for the examples [Eq. (22)]. (Although any twisted form can be written as a logarithmic form [98], it is a nontrivial question whether such a form is independent of $\Lambda$ and has no kinematic poles. This is true in pure spinor superspace [86,92].) The answer has to be proportional to $\operatorname{Pf} \boldsymbol{\Psi}_{[k \ell]}$ plus corrections polynomial in $\partial W / \partial z_{i}$ since the latter ought to vanish after taking the $\Lambda \rightarrow 0$ limit which, by Eq. (10), imposes scattering equations $d W=0$, cf. [94,95]. Finding a closed-form expression for all $n$ remains an open problem, which is of both theoretical and practical importance.

Generalization to higher-loop order consists of two separate steps. The first is writing down the analogue of Eq. (4) in terms of $(3 g+n-3)$-fold residues on genus- $g$ moduli spaces, which necessarily satisfy the kinematic Jacobi identity by the same arguments as for $g=0$, thus proving that there is no topological obstruction to imposing Eq. (2) at any loop order. The second step is finding appropriate twisted forms generalizing Eq. (13) that give rise to loop integrands of gauge and gravity theories. The latter problem needs to be considered in light of the fact that projectedness of supermoduli spaces [which was implicitly assumed in deriving Eq. (13)] breaks down at genus five [99].

The author thanks Ricardo Monteiro, Radu Roiban, and Edward Witten for many useful comments. He gratefully acknowledges the funding provided by Carl P. Feinberg. The author would like to thank Hadleigh Frost and Lionel Mason for sharing their parallel work [100] containing certain overlap with this Letter.

*smizera@ias.edu

[1] Z. Bern, J. J. M. Carrasco, and H. Johansson, New relations for gauge-theory amplitudes, Phys. Rev. D 78, 085011 (2008).

[2] Z. Bern, J. J. M. Carrasco, and H. Johansson, Perturbative Quantum Gravity as a Double Copy of Gauge Theory, Phys. Rev. Lett. 105, 061602 (2010).

[3] Z. Bern, C. Boucher-Veronneau, and H. Johansson, $\mathrm{N}>=4$ supergravity amplitudes from gauge theory at one loop, Phys. Rev. D 84, 105035 (2011).

[4] Z. Bern, J. J. M. Carrasco, L. J. Dixon, H. Johansson, and R. Roiban, Simplifying multiloop integrands and 
ultraviolet divergences of gauge theory and gravity amplitudes, Phys. Rev. D 85, 105014 (2012).

[5] Z. Bern, S. Davies, T. Dennen, and Y.-t. Huang, Absence of Three-Loop Four-Point Divergences in $N=4$ Supergravity, Phys. Rev. Lett. 108, 201301 (2012).

[6] Z. Bern, S. Davies, T. Dennen, and Y.-t. Huang, Ultraviolet cancellations in half-maximal supergravity as a consequence of the double-copy structure, Phys. Rev. D 86, 105014 (2012).

[7] Z. Bern, S. Davies, and T. Dennen, The ultraviolet critical dimension of half-maximal supergravity at three loops, arXiv:1412.2441.

[8] Z. Bern, J. J. Carrasco, W.-M. Chen, A. Edison, H. Johansson, J. Parra-Martinez, R. Roiban, and M. Zeng, Ultraviolet properties of $\mathcal{N}=8$ supergravity at five loops, Phys. Rev. D 98, 086021 (2018).

[9] R. Monteiro, D. O'Connell, and C. D. White, Black holes and the double copy, J. High Energy Phys. 12 (2014) 056.

[10] A. K. Ridgway and M. B. Wise, Static spherically symmetric Kerr-Schild metrics and implications for the classical double copy, Phys. Rev. D 94, 044023 (2016).

[11] W. D. Goldberger and A. K. Ridgway, Radiation and the classical double copy for color charges, Phys. Rev. D 95, 125010 (2017).

[12] A. Luna, R. Monteiro, I. Nicholson, A. Ochirov, D. O'Connell, N. Westerberg, and C. D. White, Perturbative spacetimes from Yang-Mills theory, J. High Energy Phys. 04 (2017) 069.

[13] N. Bahjat-Abbas, A. Luna, and C. D. White, The KerrSchild double copy in curved spacetime, J. High Energy Phys. 12 (2017) 004.

[14] J. Plefka, J. Steinhoff, and W. Wormsbecher, Effective action of dilaton gravity as the classical double copy of Yang-Mills theory, Phys. Rev. D 99, 024021 (2019).

[15] T. Adamo, E. Casali, L. Mason, and S. Nekovar, Plane wave backgrounds and colour-kinematics duality, J. High Energy Phys. 02 (2019) 198.

[16] A. Luna, R. Monteiro, I. Nicholson, and D. O'Connell, Type D spacetimes and the Weyl double copy, Classical Quantum Gravity 36, 065003 (2019).

[17] C. Cheung, I. Z. Rothstein, and M. P. Solon, From Scattering Amplitudes to Classical Potentials in the Post-Minkowskian Expansion, Phys. Rev. Lett. 121, 251101 (2018).

[18] D. A. Kosower, B. Maybee, and D. O'Connell, Amplitudes, observables, and classical scattering, J. High Energy Phys. 02 (2019) 137

[19] Z. Bern, C. Cheung, R. Roiban, C.-H. Shen, M. P. Solon, and M. Zeng, Scattering Amplitudes and the Conservative Hamiltonian for Binary Systems at Third Post-Minkowskian Order, Phys. Rev. Lett. 122, 201603 (2019).

[20] A. Antonelli, A. Buonanno, J. Steinhoff, M. van de Meent, and J. Vines, Energetics of two-body Hamiltonians in postMinkowskian gravity, Phys. Rev. D 99, 104004 (2019).

[21] Y. F. Bautista and A. Guevara, From Scattering amplitudes to classical physics: Universality, double copy and soft theorems, arXiv:1903.12419.

[22] Z. Bern, C. Cheung, R. Roiban, C.-H. Shen, M. P. Solon, and $\mathrm{M}$. Zeng, Black hole binary dynamics from the double copy and effective theory, J. High Energy Phys. 10 (2019) 206.
[23] Z. Bern, T. Dennen, Y.-t. Huang, and M. Kiermaier, Gravity as the Square of Gauge Theory, Phys. Rev. D 82, 065003 (2010).

[24] Z. Bern, J. J. M. Carrasco, L. J. Dixon, H. Johansson, and R. Roiban, The Complete Four-Loop Four-Point Amplitude in $N=4$ Super-Yang-Mills Theory, Phys. Rev. D 82, 125040 (2010).

[25] Y.-J. Du and H. Luo, On general BCJ relation at one-loop level in Yang-Mills theory, J. High Energy Phys. 01 (2013) 129.

[26] R. H. Boels, R. S. Isermann, R. Monteiro, and D. O'Connell, Colour-kinematics duality for one-loop rational amplitudes, J. High Energy Phys. 04 (2013) 107.

[27] N. E. J. Bjerrum-Bohr, T. Dennen, R. Monteiro, and D. O'Connell, Integrand oxidation and one-loop colour-dual numerators in $N=4$ gauge theory, J. High Energy Phys. 07 (2013) 092.

[28] Z. Bern, S. Davies, T. Dennen, Y.-t. Huang, and J. Nohle, Color-kinematics duality for pure yang-mills and gravity at one and two loops, Phys. Rev. D 92, 045041 (2015).

[29] A. Ochirov and P. Tourkine, BCJ duality and double copy in the closed string sector, J. High Energy Phys. 05 (2014) 136.

[30] C. R. Mafra and O. Schlotterer, Two-loop five-point amplitudes of super Yang-Mills and supergravity in pure spinor superspace, J. High Energy Phys. 10 (2015) 124.

[31] G. Mogull and D. O'Connell, Overcoming obstacles to colour-kinematics duality at two loops, J. High Energy Phys. 12 (2015) 135.

[32] S. He, R. Monteiro, and O. Schlotterer, String-inspired BCJ numerators for one-loop MHV amplitudes, J. High Energy Phys. 01 (2016) 171.

[33] S. He, O. Schlotterer, and Y. Zhang, New BCJ representations for one-loop amplitudes in gauge theories and gravity, Nucl. Phys. B930, 328 (2018).

[34] Y. Geyer and R. Monteiro, Gluons and gravitons at one loop from ambitwistor strings, J. High Energy Phys. 03 (2018) 068.

[35] Y. Geyer, R. Monteiro, and R. Stark-Muchão, Two-loop scattering amplitudes: double-forward limit and colourkinematics duality, J. High Energy Phys. 12 (2019) 049.

[36] J. Broedel and L. J. Dixon, Color-kinematics duality and double-copy construction for amplitudes from higherdimension operators, J. High Energy Phys. 10 (2012) 091.

[37] J. J. M. Carrasco, M. Chiodaroli, M. Günaydin, and R. Roiban, One-loop four-point amplitudes in pure and matter-coupled $\mathrm{N}<=4$ supergravity, J. High Energy Phys. 03 (2013) 056.

[38] H. Johansson and A. Ochirov, Pure gravities via colorkinematics duality for fundamental matter, J. High Energy Phys. 11 (2015) 046.

[39] M. Chiodaroli, M. Günaydin, H. Johansson, and R. Roiban, Scattering amplitudes in $\mathcal{N}=2$ Maxwell-Einstein and Yang-Mills/Einstein supergravity, J. High Energy Phys. 01 (2015) 081.

[40] M. Chiodaroli, M. Gunaydin, H. Johansson, and R. Roiban, Spontaneously broken Yang-Mills-Einstein supergravities as double copies, J. High Energy Phys. 06 (2017) 064. 
[41] M. Chiodaroli, M. Gunaydin, H. Johansson, and R. Roiban, Complete Construction of Magical, Symmetric and Homogeneous $N=2$ Supergravities as Double Copies of Gauge Theories, Phys. Rev. Lett. 117, 011603 (2016).

[42] H. Johansson and J. Nohle, Conformal gravity from gauge theory, arXiv:1707.02965.

[43] M. Chiodaroli, M. Gunaydin, H. Johansson, and R. Roiban, Gauged Supergravities and Spontaneous Supersymmetry Breaking from the Double Copy Construction, Phys. Rev. Lett. 120, 171601 (2018).

[44] M. Chiodaroli, M. Günaydin, H. Johansson, and R. Roiban, Non-Abelian gauged supergravities as double copies, J. High Energy Phys. 06 (2019) 099.

[45] F. Cachazo, S. He, and E. Y. Yuan, Scattering Equations and Matrices: From Einstein To Yang-Mills, DBI and NLSM, J. High Energy Phys. 07 (2015) 149.

[46] F. Cachazo, P. Cha, and S. Mizera, Extensions of theories from soft limits, J. High Energy Phys. 06 (2016) 170.

[47] Y.-J. Du and C.-H. Fu, Explicit BCJ numerators of nonlinear simga model, J. High Energy Phys. 09 (2016) 174.

[48] J. J. M. Carrasco, C. R. Mafra, and O. Schlotterer, Abelian Z-theory: NLSM amplitudes and $\alpha$ '-corrections from the open string, J. High Energy Phys. 06 (2017) 093.

[49] C. Cheung and C.-H. Shen, Symmetry for Flavor-Kinematics Duality from an Action, Phys. Rev. Lett. 118, 121601 (2017).

[50] J. J. M. Carrasco, C. R. Mafra, and O. Schlotterer, Semiabelian Z-theory: NLSM $+\phi^{3}$ from the open string, J. High Energy Phys. 08 (2017) 135.

[51] H. Elvang, M. Hadjiantonis, C. R. T. Jones, and S. Paranjape, Soft bootstrap and supersymmetry, J. High Energy Phys. 01 (2019) 195.

[52] S. Mizera and B. Skrzypek, Perturbiner methods for effective field theories and the double copy, J. High Energy Phys. 10 (2018) 018.

[53] I. Low and Z. Yin, New flavor-kinematics dualities and extensions of nonlinear sigma models, arXiv:1911.08490.

[54] R. Monteiro and D. O'Connell, The kinematic algebra from the self-dual sector, J. High Energy Phys. 07 (2011) 007.

[55] N. E. J. Bjerrum-Bohr, P. H. Damgaard, R. Monteiro, and D. O'Connell, Algebras for amplitudes, J. High Energy Phys. 06 (2012) 061.

[56] G. Chen, H. Johansson, F. Teng, and T. Wang, On the kinematic algebra for BCJ numerators beyond the MHV sector, J. High Energy Phys. 11 (2019) 055.

[57] Z. Bern, J. J. Carrasco, M. Chiodaroli, H. Johansson, and R. Roiban, The duality between color and kinematics and its applications, arXiv:1909.01358.

[58] S. Mizera, Scattering Amplitudes from Intersection Theory, Phys. Rev. Lett. 120, 141602 (2018).

[59] H. Kawai, D. C. Lewellen, and S. H. H. Tye, A relation between tree amplitudes of closed and open strings, Nucl. Phys. B269, 1 (1986).

[60] S. Mizera, Combinatorics and topology of KawaiLewellen-Tye relations, J. High Energy Phys. 08 (2017) 097.

[61] S. Mizera, Inverse of the String Theory KLT Kernel, J. High Energy Phys. 06 (2017) 084.
[62] S. Mizera, Aspects of scattering amplitudes and moduli space localization, Ph.D. thesis, Perimeter Inst. Theor. Phys., 2019.

[63] E. Plahte, Symmetry properties of dual tree-graph n-point amplitudes, Nuovo Cimento A 66, 713 (1970).

[64] E. Casali, S. Mizera, and P. Tourkine, Monodromy relations from twisted homology, J. High Energy Phys. 12 (2019) 087.

[65] P. Mastrolia and S. Mizera, Feynman integrals and intersection theory, J. High Energy Phys. 02 (2019) 139.

[66] H. Frellesvig, F. Gasparotto, S. Laporta, M. K. Mandal, P. Mastrolia, L. Mattiazzi, and S. Mizera, Decomposition of Feynman integrals on the maximal cut by intersection numbers, J. High Energy Phys. 05 (2019) 153.

[67] H. Frellesvig, F. Gasparotto, M. K. Mandal, P. Mastrolia, L. Mattiazzi, and S. Mizera, Vector Space of Feynman Integrals and Multivariate Intersection Numbers, Phys. Rev. Lett. 123, 201602 (2019).

[68] S. Mizera and A. Pokraka, From infinity to four dimensions: Higher residue pairings and Feynman integrals, J. High Energy Phys. 02 (2020) 159.

[69] L. de la Cruz, A. Kniss, and S. Weinzierl, Properties of scattering forms and their relation to associahedra, J. High Energy Phys. 03 (2018) 064.

[70] H. Frost, Biadjoint scalar tree amplitudes and intersecting dual associahedra, J. High Energy Phys. 06 (2018) 153.

[71] Z. Li and C. Zhang, Moduli space of paired punctures, cyclohedra and particle pairs on a circle, J. High Energy Phys. 05 (2019) 029.

[72] F. Brown and C. Dupont, Lauricella hypergeometric functions, unipotent fundamental groups of the punctured Riemann sphere, and their motivic coactions, arXiv:1907.06603.

[73] F. Brown and C. Dupont, Single-valued integration and superstring amplitudes in genus zero, arXiv:1910.01107.

[74] S. Abreu, R. Britto, C. Duhr, E. Gardi, and J. Matthew, From positive geometries to a coaction on hypergeometric functions, J. High Energy Phys. 02 (2020) 122.

[75] N. Kalyanapuram, Stokes polytopes and intersection theory, arXiv:1910.12195.

[76] P. Deligne and D. Mumford, The irreducibility of the space of curves of given genus, Publ. Math. Inst. Hautes Étud. Sci. 36, 75 (1969).

[77] F. Cachazo, L. Mason, and D. Skinner, Gravity in twistor space and its Grassmannian formulation, SIGMA 10, 051 (2014).

[78] F. C. S. Brown, Multiple zeta values and periods of moduli spaces $\overline{\mathfrak{M}}_{0, n}(\mathbb{R})$, Ann. Sci. Ecole Norm. Sup. 42, 371 (2009).

[79] N. Arkani-Hamed, Y. Bai, S. He, and G. Yan, Scattering forms and the positive geometry of kinematics, color and the worldsheet, J. High Energy Phys. 05 (2018) 096.

[80] V. I. Arnol'd, The cohomology ring of the colored braid group, Mathematical notes of the Academy of Sciences of the USSR 5, 138 (1969).

[81] K. Aomoto, Gauss-Manin connection of integral of difference products, J. Math. Soc. Jpn. 39, 191 (1987).

[82] P. Deligne and G. Mostow, Monodromy of hypergeometric functions and non-lattice integral monodromy, Publ. Math. Inst. Hautes Étud. Sci. 63, 5 (1986). 
[83] K. Cho and K. Matsumoto, Intersection theory for twisted cohomologies and twisted Riemann's period relations I, Nagoya mathematical Journal 139, 67 (1995).

[84] K. Matsumoto, Intersection numbers for logarithmic $k$ forms, Osaka Journal of mathematics 35, 873 (1998).

[85] M. Green, J. Schwarz, and E. Witten, in Superstring Theory: Volume 1, Introduction, Cambridge Monographs on Mathematical Physics (Cambridge University Press, Cambridge, England, 1988).

[86] C. R. Mafra, O. Schlotterer, and S. Stieberger, Complete $\mathrm{N}$-point superstring disk amplitude I. Pure spinor computation, Nucl. Phys. B873, 419 (2013).

[87] F. Cachazo, Fundamental BCJ relation in $N=4$ SYM from the connected formulation, arXiv:1206.5970.

[88] F. Cachazo, S. He, and E. Y. Yuan, Scattering of massless particles: Scalars, gluons and gravitons, J. High Energy Phys. 07 (2014) 033.

[89] T. Azevedo, M. Chiodaroli, H. Johansson, and O. Schlotterer, Heterotic and bosonic string amplitudes via field theory, J. High Energy Phys. 10 (2018) 012.

[90] F. Cachazo, S. He, and E. Y. Yuan, Scattering of Massless Particles in Arbitrary Dimensions, Phys. Rev. Lett. 113, 171601 (2014).

[91] K. Saito, The higher residue pairings $K_{F}^{(k)}$ for a family of hypersurface singular points, in Singularities, Part 2
(Arcata, Calif., 1981), Proc. Sympos. Pure Math. Vol. 40 (Amer. Math. Soc., Providence, RI, 1983), pp. 441-463.

[92] C. R. Mafra, O. Schlotterer, and S. Stieberger, Explicit BCJ numerators from pure spinors, J. High Energy Phys. 07 (2011) 092.

[93] O. Schlotterer, Amplitude relations in heterotic string theory and Einstein-Yang-Mills, J. High Energy Phys. 11 (2016) 074.

[94] C.-H. Fu, Y.-J. Du, R. Huang, and B. Feng, Expansion of Einstein-Yang-Mills amplitude, J. High Energy Phys. 09 (2017) 021.

[95] Y.-J. Du and F. Teng, BCJ numerators from reduced Pfaffian, J. High Energy Phys. 04 (2017) 033.

[96] S. He, F. Teng, and Y. Zhang, String Amplitudes from Field-Theory Amplitudes and Vice Versa, Phys. Rev. Lett. 122, 211603 (2019).

[97] S. He, F. Teng, and Y. Zhang, String correlators: Recursive expansion, integration-by-parts and scattering equations, J. High Energy Phys. 09 (2019) 085.

[98] K. Saito, Theory of logarithmic differential forms and logarithmic vector fields, J. Fac. Sci. Univ. Tokyo Sect. IA Math 27, 265 (1980).

[99] R. Donagi and E. Witten, Supermoduli space is not projected, Proc. Symp. Pure Math. 90, 19 (2015).

[100] H. Frost and L. Mason, Lie polynomials and a twistorial correspondence for amplitudes, arXiv:1912.04198. 